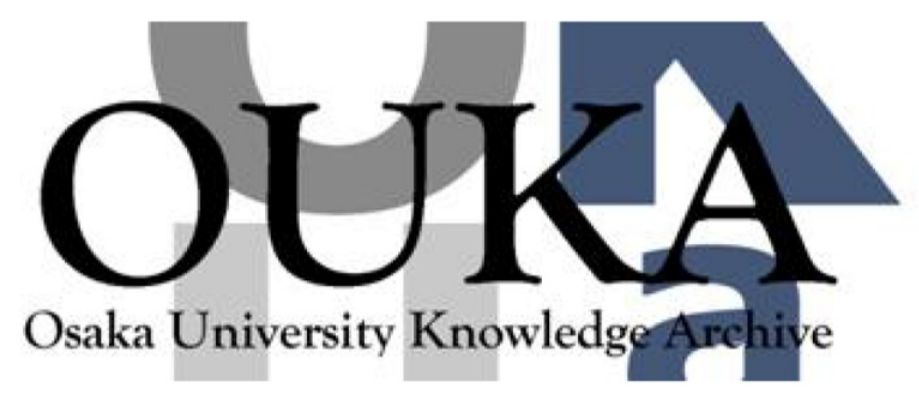

\begin{tabular}{|c|l|}
\hline Title & $\begin{array}{l}\text { Theoretical study of transition radiation from } \\
\text { hot electrons generated in the laser-solid } \\
\text { interaction }\end{array}$ \\
\hline Author(s) & Zheng, Jian; Tanaka, K. A. ; Miyakoshi, T. et al. \\
\hline Citation & Physics of Plasmas. 10(7) p. 2994-p. 3003 \\
\hline Issue Date & $2003-07$ \\
\hline oaire:version & VoR \\
\hline URL & https://hdl. handle. net/11094/3009 \\
\hline rights & \\
\hline Note & \\
\hline
\end{tabular}

Osaka University Knowledge Archive : OUKA

https://ir. Library. osaka-u. ac. jp/

Osaka University 


\title{
Theoretical study of transition radiation from hot electrons generated in the laser-solid interaction
}

\author{
Jian Zheng, ${ }^{\text {a) }}$ K. A. Tanaka, ${ }^{\text {b) }}$ T. Miyakoshi, Y. Kitagawa, R. Kodama, T. Kurahashi, \\ and T. Yamanaka \\ Institute of Laser Engineering, Osaka University, 2-6 Yamada-oka, Suita, Osaka 565-0871, Japan
}

(Received 5 February 2003; accepted 27 March 2003)

\begin{abstract}
Transition radiation from a beam of hot electrons generated in ultraintense laser plasma interaction is theoretically studied. The total radiation is separated into two parts: one is incoherent transition radiation (ITR), the other is coherent transition radiation (CTR). The spectrum of ITR just depends on the particle velocity distribution in the beam. The angular distribution of ITR varies from $\sin ^{2} \theta$, and approaches the angular distribution of the beam when the hot electron temperature increases from the nonrelativistic limit $\left(T \ll m c^{2}\right)$ to the ultrarelativistic limit $\left(T \gg m c^{2}\right)$. The spectrum of CTR is dependent on the particle configuration as well as their velocities. Any microbunching in the beam can greatly enhance the CTR intensity at the microbunching frequency, from which the dominant heating process can be inferred. The effects of target thickness and hot electron temperature on CTR intensity are also calculated. The simplified model shows that the CTR intensity decreases with the increase of the target thickness, and increases with the hot electron temperature. The divergence of the beam can broaden the CTR spectrum. () 2003 American Institute of Physics. [DOI: 10.1063/1.1576388]
\end{abstract}

\section{INTRODUCTION}

Recently, there has been an increasing interest in energetic (hot) electrons generated in ultraintense laser plasma interactions because of their many potential applications in various fields, such as plasma accelerators, ${ }^{1,2}$ fast ignition, ${ }^{3,4}$ and positron-electron plasmas. ${ }^{5,6} \mathrm{Up}$ to now, many heating mechanisms have been proposed for the generation of these hot electrons in laser plasma interactions: stimulated Raman scattering, ${ }^{7}$ vacuum heating, ${ }^{8} \quad \mathbf{j} \times \mathbf{B}$ heating, ${ }^{9}$ betatron acceleration, ${ }^{10}$ and many others. Hot electrons have been studied by various methods: directly measuring electron energy spectra by using an electron spectrometer, ${ }^{2,11,12}$ or indirectly by detecting $K \alpha$ x-ray emission, ${ }^{13,14}$ or bremsstrahlung $\mathrm{x}$-ray emission. ${ }^{14-16}$ These measurements can provide valuable information on the hot electrons, such as the electron energy spectrum, electron number, and electron beam divergence. Occasionally, we need more information to confirm which heating mechanism is the dominant process in a certain experiment. The bunch form of a beam of hot electrons could be direct evidence of the dominant heating process because the hot electrons acquire characteristic microbunching in different heating process. For example, electrons are accelerated once in the forward direction in one laser circle in vacuum heating, ${ }^{8}$ but twice in one laser circle in $\mathbf{j} \times \mathbf{B}$ heating. ${ }^{9}$ Therefore, the hot electrons generated in $\mathbf{j}$ $\times \mathbf{B}$ heating should have a different bunch form from those produced in vacuum heating. If we can measure the mi-

\footnotetext{
a) Present address: Department of Modern Physics, University of Science and Technology of China, Hefei, Anhui 230027, People's Republic of China; electronic mail: jzheng@ustc.edu.cn

b) Also at the Department of Electromagnetic Energy Engineering, Graduate School of Engineering, Osaka University, 2-1 Yamada-oka, Suita, Osaka 565-0871, Japan.
}

crobunch of hot electrons, we can definitely know the dominant heating process. Unfortunately, the methods mentioned above cannot give us such information. In this respect, a radiation phenomenon, i.e., transition radiation, can provide an approach to the detailed measurement of hot electrons.

Transition radiation is a radiation phenomenon in which electromagnetic waves are emitted when a charged particle moves across the interface between two media with different dielectric constants. ${ }^{17,18}$ Transition radiation has been extensively studied by using accelerators. It has been studied in the $\mathrm{x}$-ray region, ${ }^{19-21}$ the far-infrared region, ${ }^{22-24}$ and the optical region. ${ }^{26-28}$ Coherent transition radiation is more attractive because it can provide valuable detailed information on electron beams. Coherent transition radiation has been experimentally studied in the far-infrared region, ${ }^{22-25}$ and in the optical region. ${ }^{27-29}$ By detecting coherent transition radiation, the characteristics of electron beams, such as their three-dimensional distributions and the divergence of electrons in a bunch, ${ }^{24}$ and electron-beam microbunching, ${ }^{25,28,29}$ have been measured. Very recently, Baton et al. reported their first measurement of coherent transition radiation from hot electrons generated in laser solid interactions. ${ }^{30}$ However, hot electrons generated in laser plasma interaction have properties different from those in accelerators. Usually, the electrons in an accelerator are highly collimated and monochromatic. Hot electrons produced in laser plasma interactions have a divergence angle, and a Boltzmann energy distribution. Therefore, it is necessary to develop transition radiation theory to include the case of hot electrons. We have theoretically studied transition radiation in the condition relevant to hot electrons generated in ultraintense laser plasma interactions. ${ }^{31}$ In that paper, we consider the case that hot electrons are collimated, and move along the target normal. 


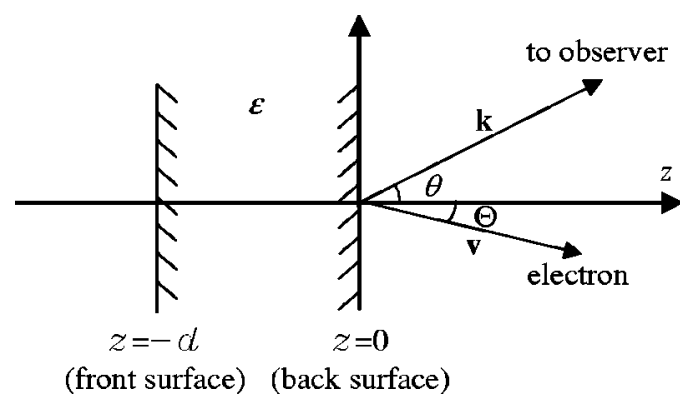

FIG. 1. Configuration of the calculation.

In this article, we extend our previous research to more realistic conditions. For the sake of simplification, however, some physics is not included in our calculations, such as the influences of self-generated magnetic field, and small angle scattering of hot electrons inside the dense plasma. In Sec. II, we derive the formulas of transition radiation. In Secs. III and IV, we discuss the spectra of incoherent and coherent transition radiation. Finally, we draw conclusions in Sec. V.

\section{EQUATIONS OF TRANSITION RADIATION}

We consider transition radiation from a beam of $N$ electrons passing through a target foil. The configuration of our calculation is shown in Fig. 1. As seen in this figure, the rear surface of the target locates at the plane of $z=0$, and the front surface locates at the plane of $z=-d$, where $d$ is the target thickness. The ambient is vacuum. Hot electrons generated at the front surface move from the left to the right. The origin of the coordinate system of our calculation is the point where the beam center crosses the rear surface. We decompose the particle velocity $\mathbf{v}$, the particle coordinate $\mathbf{r}$, and the wave vector $\mathbf{k}$ of the radiation into the tangential and normal components: $\mathbf{v}=(\mathbf{w}, u)=\left(v_{x}, v_{y}, v_{z}\right), \quad \mathbf{r}=(\boldsymbol{\rho}, z)$ $=(x, y, z)$, and $\mathbf{k}=(\mathbf{q}, \eta)=\left(k_{x}, k_{y}, k_{z}\right)$. The particle moving direction and the radiation emission direction are described by the two sets of the angles, $(\Theta, \Phi)$ and $(\theta, \phi)$, respectively. With these angles, the particle velocity can be written as $\mathbf{v}$ $=v(\sin \Theta \cos \Phi, \sin \Theta \sin \Phi, \cos \Theta)$, and the wave vector of the radiation as $\mathbf{k}=k(\sin \theta \cos \phi, \sin \theta \sin \phi, \cos \theta)$. In what follows, we derive the formulas of the transition radiation into the vacuum $(z>0)$ as hot electrons pass through the rear surface of the target.

We start our calculation from the set of the equations, ${ }^{32}$

$$
\begin{aligned}
& \nabla^{2} \mathbf{A}-\frac{\hat{\epsilon}}{c^{2}} \frac{\partial^{2}}{\partial t^{2}} \mathbf{A}=-\frac{4 \pi}{c} e \sum_{i=1}^{N} \mathbf{v}_{i} \delta\left[z-u_{i}\left(t-t_{i}\right)\right] \\
& \times \delta\left[\boldsymbol{\rho}-\boldsymbol{\rho}_{i}-\mathbf{w}_{i}\left(t-t_{i}\right)\right], \\
& \hat{\epsilon}\left(\nabla^{2} \varphi-\frac{\hat{\epsilon}}{c^{2}} \frac{\partial^{2}}{\partial t^{2}} \varphi\right)=-4 \pi e \sum_{i=1}^{N} \mathbf{v}_{i} \delta\left[z-u_{i}\left(t-t_{i}\right)\right] \\
& \times \delta\left[\boldsymbol{\rho}-\boldsymbol{\rho}_{i}-\mathbf{w}_{i}\left(t-t_{i}\right)\right],
\end{aligned}
$$

where $\mathbf{A}$ and $\varphi$ are the vector and scalar potentials of the electromagnetic fields, $\delta(\cdots)$ is the $\delta$-function, $c$ is the light speed, $t_{i}$ is the time when the $i$ th electron crosses the rear surface, $\mathbf{v}_{i}=\left(\mathbf{w}_{i}, u_{i}\right)$ is the velocity of the $i$ th electron, $\boldsymbol{\rho}_{i}$ is the tangential coordinate of the $i$ th electron at the time $t_{i}, \hat{\epsilon}$ is an operator defined by $\hat{\epsilon} \exp (-i \omega t)=\epsilon(\omega) \exp (-i \omega t),{ }^{32}$ and $\epsilon(\omega)$ is the dielectric function of the target material. Here, we do not consider the magnetic properties of the target material, and simply set the magnetic permeability equal to 1 . Since radiation field is transverse, and satisfies the dispersion relation $k^{2}=\epsilon \omega^{2} / c^{2}$, where $\omega$ is the radiation frequency, the Fourier component of an radiation field can be written as

$$
\mathbf{E}^{r}(\mathbf{k}, \omega)=\mathfrak{E}_{z}^{r}\left[\hat{\mathbf{z}}-\left(\eta / q^{2}\right) \mathbf{q}\right] \delta\left(k^{2}-\epsilon \omega^{2} / c^{2}\right),
$$

where $\hat{\mathbf{z}}$ is the unit vector along the $z$-axis, $\delta\left(k^{2}-\epsilon \omega^{2} / c^{2}\right)$ is the $\delta$-function, and $\mathfrak{E}_{z}^{r}$ is the amplitude of the field. Following the method developed in Ref. 33, we obtain $\mathfrak{E}_{z}^{r}$ in the vacuum $(z>0)$,

$$
\mathfrak{E}_{z}^{r}=\sum_{i=1}^{N} \mathfrak{E}_{i}\left(\mathbf{w}_{i}, u_{i}\right) e^{i \omega t_{i}-i \mathbf{q} \cdot \boldsymbol{\rho}_{i}},
$$

where

$$
\begin{aligned}
\mathfrak{E}_{i}= & -i \frac{4(2 \pi)^{2} e\left|\eta_{2}\right|}{\left(\left|\eta_{1}\right|+\epsilon\left|\eta_{2}\right|\right) u_{i}} \\
& \times\left\{-\frac{\left(q^{2}-\epsilon \omega \mathbf{q} \cdot \mathbf{w}_{i} / c^{2}\right)\left(1+\left|\eta_{1}\right| / \kappa_{i}\right)}{q^{2}+\kappa_{i}^{2}-\epsilon(\omega / c)^{2}}\right. \\
& \left.+\frac{\left(q^{2}-\omega \mathbf{q} \cdot \mathbf{w}_{i} / c^{2}\right)\left(\epsilon+\left|\eta_{1}\right| / \kappa_{i}\right)}{q^{2}+\kappa_{i}^{2}-(\omega / c)^{2}}\right\},
\end{aligned}
$$

and

$$
\begin{aligned}
& \eta_{1}=-\sqrt{\epsilon \omega^{2} / c^{2}-q^{2}}, \quad \eta_{2}=\sqrt{\omega^{2} / c^{2}-q^{2}}, \\
& \kappa_{i}=\left(\omega-\mathbf{q} \cdot \mathbf{w}_{i}\right) / u_{i} .
\end{aligned}
$$

The energy spectrum of transition radiation into the solid angle $d \Omega$ is then given by

$$
\frac{d^{2} \mathcal{E}}{d \omega d \Omega}=\frac{c}{4(2 \pi)^{6} \sin ^{2} \theta}\left|\sum_{i=1}^{N} \mathfrak{E}_{i}\left(\mathbf{w}_{i}, u_{i}\right) e^{i \omega t_{i}-i \mathbf{q} \cdot \boldsymbol{\rho}_{i}}\right|^{2},
$$

where $\omega \geqslant 0$.

We separate the spectrum of transition radiation (6) into two parts: the spectrum of incoherent transition radiation (ITR) and the spectrum of coherent transition radiation (CTR). The former is the summation of the radiation spectra from individual particles, and the latter is governed by the interference between the radiation waves from different charges. The ITR spectrum is given by

$$
\frac{d^{2} \mathcal{E}_{\text {ITR }}}{d \omega d \Omega}=\frac{c}{4(2 \pi)^{6} \sin ^{2} \theta} \sum_{i=1}^{N}\left|\mathfrak{E}_{i}\left(\mathbf{w}_{i}, u_{i}\right)\right|^{2},
$$


and the CTR spectrum is given by

$$
\begin{aligned}
\frac{d^{2} \mathcal{E}_{\mathrm{CTR}}}{d \omega d \Omega}= & \frac{c}{4(2 \pi)^{6} \sin ^{2} \theta} \sum_{\substack{i, j=1 \\
(i \neq j)}}^{N} \mathfrak{E}_{i}\left(\mathbf{w}_{i}, u_{i}\right) \mathfrak{E}_{j}^{*}\left(\mathbf{w}_{j}, u_{j}\right) \\
& \times e^{i \omega\left(t_{i}-t_{j}\right)-i \mathbf{q} \cdot\left(\boldsymbol{\rho}_{i}-\boldsymbol{\rho}_{j}\right)} .
\end{aligned}
$$

Equation (4) becomes rather lengthy after substituting Eq. (5) into it. This equation can be simplified if the target is made of good conductor like aluminum and silver. In this case, we have $|\epsilon| \gg 1$ in the optical and lower frequency region. Letting $|\epsilon| \rightarrow \infty$, Eq. (4) is simplified,

$$
\mathfrak{E}_{i}=-i \frac{4(2 \pi)^{2} e \beta_{i}}{c} \frac{\sin \theta \cos \Theta_{i}\left[\sin \theta-\beta_{i} \sin \theta \cos \left(\phi-\Phi_{i}\right)\right]}{\left[\left(1-\beta_{i} \sin \theta \sin \Theta_{i} \cos \left(\phi-\Phi_{i}\right)\right)^{2}-\beta_{i}^{2} \cos ^{2} \theta \cos ^{2} \Theta_{i}\right]},
$$

where $\beta_{i}=v_{i} / c$ is the normalized particle velocity, and $\left(\Theta_{i}, \Phi_{i}\right)$ is the particle moving direction. In the limit of $|\epsilon| \rightarrow \infty$, transition radiation can be considered as the radiation due to the creation (annihilation) of an electric dipole consisting of the electron and its image charge when an electron leaves (impacts) the conductor surface.

Before the discussions of transition radiation from an electron beam, we briefly discuss it from single particle. For a single electron, the transition radiation spectrum is given by

$$
\frac{d^{2} \mathcal{E}_{\text {single }}}{d \omega d \Omega}=\frac{e^{2}}{\pi^{2} c} \frac{\beta^{2} \cos ^{2} \Theta[\sin \theta-\beta \sin \Theta \cos (\phi-\Phi)]^{2}}{\left[(1-\beta \sin \theta \sin \Theta \cos (\phi-\Phi))^{2}-\beta^{2} \cos ^{2} \theta \cos ^{2} \Theta\right]^{2}} .
$$

If the electron just moves in the plane perpendicular to the $z$-axis, i.e., $\Theta=90^{\circ}$, transition radiation vanishes. This result is natural because the electron does not cross the interface. The intensity of transition radiation becomes zero at the emission angle,

$$
\theta_{0}=\arcsin [\beta \sin \Theta \cos (\phi-\Phi)] .
$$

When $\phi=\Phi$, we have $\theta_{0}=\arcsin (\beta \sin \Theta)$. In the ultrarelativistic case, this angle approaches $\Theta$. In the cross plane of $\phi=\Phi$, the intensity of transition radiation has two maxima around the angle $\theta_{0}$, which are located at

$$
\theta_{\max }=\arcsin \left(\beta \sin \Theta \pm \sqrt{(\beta-1 / \beta)^{2}+\left(1-\beta^{2}\right) \cos ^{2} \Theta}\right) .
$$

When $\beta \rightarrow 1$, we also have $\theta_{\max } \rightarrow \Theta$. Therefore, transition radiation is emitted within a small angle along the direction of the moving charge in the ultrarelativistic case. In Fig. 2, we plot in the cross plane of $\phi=\Phi$ the angular distribution of transition radiation from a single electron with various

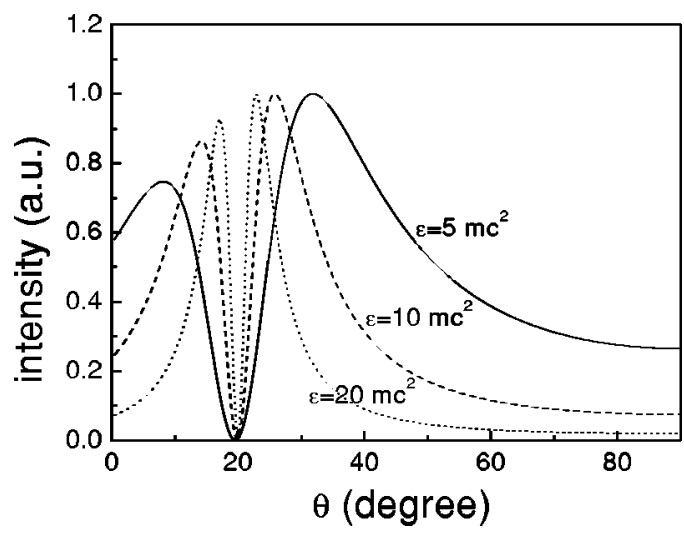

FIG. 2. Angular distribution of transition radiation in the cross plane of $\phi$ $=\Phi$ from single electron with different energies, where the moving direction of the electron is $(\Theta, \Phi)=\left(20^{\circ}, 180^{\circ}\right)$. energies, whose moving angle is $(\Theta, \Phi)=\left(20^{\circ}, 180^{\circ}\right)$. As seen in this figure, the radiation vanishes near the angle of $\theta=20^{\circ}$. And as the electron energy increases, the angles of the two maxima move towards $20^{\circ}$. In the nonrelativistic case, we approximately have

$$
\frac{d^{2} \mathcal{E}_{\text {single }}}{d \omega d \Omega}=\frac{e^{2}}{\pi^{2} c} \beta^{2} \cos ^{2} \Theta \sin ^{2} \theta .
$$

The radiation energy is proportional to the electron kinetic energy, and its angular distribution is described by $\sin ^{2} \theta$. This results is the same with the radiation from an electric dipole in the nonrelativistic case.

In the following two sections, we discuss the spectra of ITR and CTR based on Eqs. (7), (8), and (9).

\section{INCOHERENT TRANSITION RADIATION}

The ITR spectrum is the summation of radiation spectra from individual electrons. It depends only on the velocities of the electrons of concern. Since the number of hot electrons generated in ultraintense laser plasma interactions is usually very large, we can describe the hot electrons quite well with a velocity distribution function, which is defined as the average of the exact velocity distribution,

$$
f_{\mathbf{v}}(\mathbf{v})=\left\langle\frac{1}{N} \sum_{i=1}^{N} \delta\left(\mathbf{v}-\mathbf{v}_{i}\right)\right\rangle
$$

where the average $\langle\cdots\rangle$ is taken over physical infinitesimal volume in velocity space that still contains many electrons. The average in Eq. (11) can also be considered as an ensemble average. ${ }^{34}$ In this case, we image that an infinite series of $\mathrm{N}$-electron beams is created under identical conditions.

With the aid of the velocity distribution function, we can replace the summation in Eq. (7) with the integral, 


$$
\frac{d^{2} \mathcal{E}_{\text {ITR }}}{d \omega d \Omega}=\frac{c N}{4(2 \pi)^{6} \sin ^{2} \theta} \int d \mathbf{v} f_{\mathbf{v}}(\mathbf{v})|\mathfrak{E}(\mathbf{w}, u)|^{2} .
$$

Substituting Eq. (9) into Eq. (12), the ITR spectrum is now given by

$$
\frac{d^{2} \mathcal{E}_{\mathrm{ITR}}}{d \omega d \Omega}=\frac{e^{2} N}{\pi^{2} c} \int d \mathbf{v} \frac{\beta^{2} \cos ^{2} \Theta[\sin \theta-\beta \sin \Theta \cos (\phi-\Phi)]^{2} f_{\mathbf{v}}(\mathbf{v})}{\left[(1-\beta \sin \theta \sin \Theta \cos (\phi-\Phi))^{2}-\beta^{2} \cos ^{2} \theta \cos ^{2} \Theta\right]^{2}} .
$$

As seen in Eq. (13), the frequency spectrum of ITR is independent of the radiation frequency. This result means that the ITR spectrum is flat when the condition $|\epsilon| \gg 1$ is satisfied.

In Eq. (12), the ITR spectrum is written as a functional of the velocity distribution function of hot electrons. However, what we can measure in actual experiment is the energy distribution function. ${ }^{11}$ Using the relation between particle velocity and energy, Eq. (13) can be written as

$$
\frac{d^{2} \mathcal{E}_{\text {ITR }}}{d \omega d \Omega}=\frac{e^{2} N}{\pi^{2} c} \int d \varepsilon d \Theta d \Phi \frac{\beta^{2} \sin \Theta \cos ^{2} \Theta[\sin \theta-\beta \sin \Theta \cos (\phi-\Phi)]^{2} f_{\varepsilon}(\varepsilon, \Theta, \Phi)}{\left[(1-\beta \sin \theta \sin \Theta \cos (\phi-\Phi))^{2}-\beta^{2} \cos ^{2} \theta \cos ^{2} \Theta\right]^{2}},
$$

where $\varepsilon=\left(1-\beta^{2}\right)^{-1 / 2}-1$ is the electron kinetic energy normalized with $m c^{2}$. Both simulations ${ }^{9}$ and experiments ${ }^{11}$ show that hot electrons generated in ultraintense laser plasma interactions have approximately a Boltzmann energy distribution, i.e.,

$$
f_{\varepsilon}(\varepsilon, \Theta, \Phi) \propto e^{-\varepsilon / T},
$$

where $T$ is the hot electron temperature normalized with $m c^{2}$. Usually, the angular distribution of hot electrons is complicated. Here, for the purpose of analytical calculation, we assume that hot electron beam is symmetric about the $z$-axis, and diverges as $\cos ^{2} \Theta$. Under these assumptions, the function $f_{\varepsilon}(\varepsilon, \Theta, \Phi)$ is given by

$$
f_{\varepsilon}(\varepsilon, \Theta, \Phi)=\frac{3}{2 \pi T} e^{-\varepsilon / T} \cos ^{2} \Theta .
$$

Substituting the distribution function (15) into Eq. (14), and integrating over $\Theta$ and $\Phi$, we obtain the angular distribution of ITR spectrum,

$$
\begin{aligned}
\frac{d^{2} \mathcal{E}_{\text {ITR }}}{d \omega d \Omega}= & \frac{3 e^{2} N}{4 \pi^{2} T c} \int_{0}^{\infty} d \varepsilon \frac{\exp (-\varepsilon / T)}{\beta^{3}} \\
& \times\left\{\left[3\left(\beta^{2}-1\right)+\left(\frac{11}{2}-\frac{9}{2} \beta^{2}\right) \cos ^{2} \theta\right] \ln \frac{1+\beta}{1-\beta}\right. \\
& \left.+\frac{\beta}{3}\left(18+16 \beta^{2} \cos ^{2} \theta-12 \beta^{2}-33 \cos ^{2} \theta\right)\right\} .
\end{aligned}
$$

We further integrate Eq. (16) over the solid angle $d \Omega$ in the forward half space, and then obtain the ITR energy in the frequency interval $d \omega$,

$$
\begin{aligned}
\frac{d \mathcal{E}_{\text {ITR }}}{d \omega}= & \frac{3 e^{2} N}{2 \pi T c} \int_{0}^{\infty} d \varepsilon \frac{\exp (-\varepsilon / T)}{\beta^{3}} \\
& \times\left[\left(\frac{3}{2} \beta^{2}-\frac{7}{6}\right) \ln \frac{1+\beta}{1-\beta}+\frac{\beta}{3}\left(7-\frac{20}{3} \beta^{2}\right)\right] .
\end{aligned}
$$

The angular distribution of ITR depends on the hot electron temperature $T$, as seen from Eq. (16). When the temperature is extremely high, most electrons have velocities close to the light speed. Equation (16) is then dominated by the term proportional to $\ln [(1+\beta) /(1-\beta)]$. Letting $\beta=1$ in Eq. (16), and noticing that $\ln [(1+\beta) /(1-\beta)] \approx 2 \ln 2(1+\varepsilon)$ when $\beta \rightarrow 1$, we approximately have

$$
\begin{aligned}
\frac{d^{2} \mathcal{E}_{\text {ITR }}}{d \omega d \Omega} & \sim \frac{3 e^{2} N}{2 \pi^{2} T c} \int_{0}^{\infty} d \varepsilon \exp (-\varepsilon / T) \ln [2(1+\varepsilon)] \cos ^{2} \theta \\
& \propto \cos ^{2} \theta .
\end{aligned}
$$

We can see that when $T \gg 1$ the angular distribution of ITR approaches $\cos ^{2} \theta$, the same with that of the electron beam. This result is quite understandable. We know from the single particle theory that transition radiation from a single charge is emitted within a small angle around the charge moving direction when the charge is ultrarelativistic. As a consequence, the angular distribution of ITR is close to that of particle beam because the ITR spectrum is the summation of transition radiation spectra from individual particles.

In the nonrelativistic case, most electrons have velocities far slower than the light speed, i.e., $\beta \ll 1$. We expand the integrand in Eq. (16) in a power series of $\beta$, and just keep the lowest order term. We obtain

$$
\frac{d^{2} \mathcal{E}_{\text {ITR }}}{d \omega d \Omega} \sim \frac{3 e^{2} N}{5 \pi^{2} T c} \int_{0}^{\infty} d \varepsilon \exp (-\varepsilon / T) \beta^{2} \sin ^{2} \theta \propto \sin ^{2} \theta .
$$

The angular distribution of ITR becomes $\sin ^{2} \theta$, independent of that of the hot electron beam when the beam energy is nonrelativistic.

The above discussions show that the angular distribution of ITR from a beam of electrons described by the distribution function (15) varies from $\sin ^{2} \theta$ to $\cos ^{2} \theta$ as the temperature increases from $T \ll 1$ to $T \gg 1$. It is straightforward to imagine that the angular distribution could become flat when the temperature is appropriate. Figure 3 shows the angular distribution of the ITR from a beam of hot electrons with various temperatures. As seen from this figure, when the temperature is very low, or extremely high, the angular distribution approaches $\sin ^{2} \theta$ or $\cos ^{2} \theta$, respectively, as we predict. In the temperature range from $2 \mathrm{MeV}$ to $4 \mathrm{MeV}$, the angular distribution of the ITR is nearly flat. 


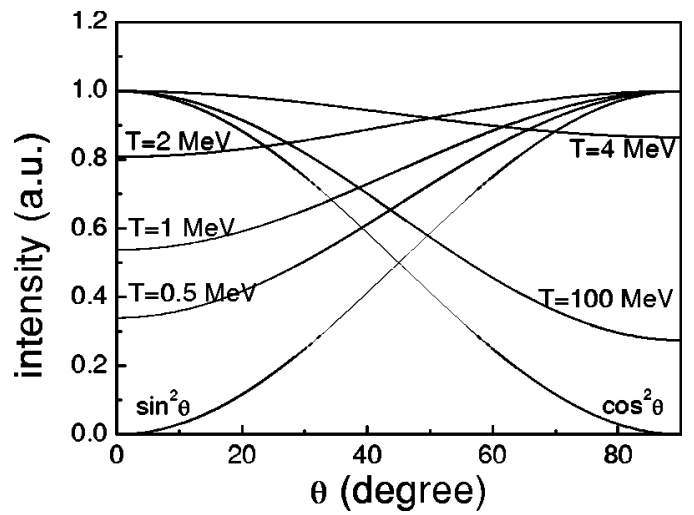

FIG. 3. Angular distribution of incoherent transition radiation from a beam of hot electrons with various temperatures, where the distribution function of hot electrons is given by Eq. (15).

The ITR energy increases with the increase of the temperature $T$. When the electron is nonrelativistic, the radiation energy from a single particle is proportional to its kinetic energy. Hence, the total radiation energy is proportional to the hot electron temperature $T$ after averaging over the Boltzmann energy distribution. When the electrons are ultrarelativistic, the radiation energy from a single particle is a logarithm function of its energy, see Eq. (18). Hence the radiation energy increases slowly with the increase of hot electron temperature in the very high temperature region. Presented in Fig. 4 is the variation of the quantity $d \mathcal{E}_{\text {ITR }} / N d \omega$ with the temperature $T$. When the temperature is $1 \mathrm{MeV}$, this quantity is about $3 \times 10^{-30} \mathrm{erg}$ s. Hence the average radiation energy from one electron into the solid angle $\Delta \Omega$ and the frequency interval $\Delta \omega$ is roughly given by

$$
\mathcal{E}_{\text {ITR }} / N \sim 3 \times 10^{-30} \Delta \omega(\Delta \Omega / 2 \pi) .
$$

As an example, the radiation energy per hot electron is about $6 \times 10^{-19}$ erg into an $\mathrm{f} / 5$ detection system with a $10-\mathrm{nm}$

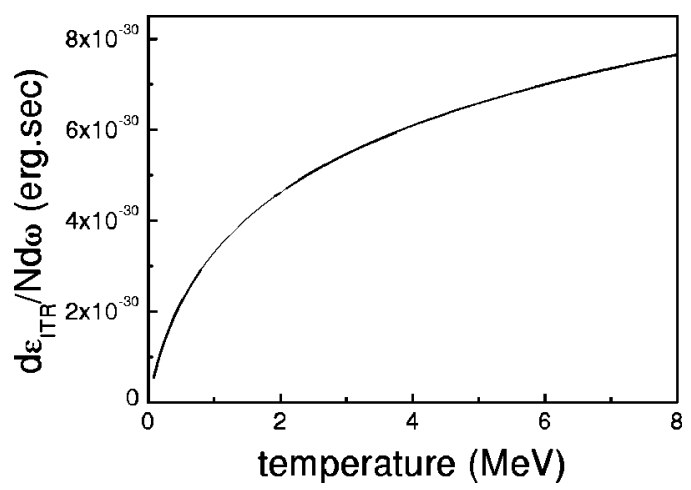

FIG. 4. Dependence of the energy of incoherent transition radiation on the hot electron temperature, where the hot electrons are described by the distribution function (15)

bandpass filter centering at $700 \mathrm{~nm}$. This means that about $5 \times 10^{6}$ hot electrons can generate one photon in the wavelength range of detection. The yield of ITR is very low. Hence it could be difficult to measure ITR in an experiment because other radiation process, like blackbody radiation from the heated target, may have a similar level in the wavelength of interest.

\section{COHERENT TRANSITION RADIATION}

The spectrum of CTR is more complex in comparison with that of ITR. It depends not only on the particle velocities but also on the particle configuration. As we did in Sec. III, we introduce a new distribution function $f_{\tau}(\tau, \boldsymbol{\rho}, \mathbf{v})$, which is defined by

$$
\begin{aligned}
& f_{\tau}(\tau, \boldsymbol{\rho}, \mathbf{v}) \\
& \quad=\left\langle\frac{1}{N} \sum_{i=1}^{N} \delta\left(\tau-t_{i}\right) \delta\left(\boldsymbol{\rho}-\boldsymbol{\rho}_{i}\right) \delta\left(\mathbf{w}-\mathbf{w}_{i}\right) \delta\left(u-u_{i}\right)\right\rangle .
\end{aligned}
$$

Substituting Eq. (9) into Eq. (8), and replacing the summation with an integral, the CTR spectrum is now given by

$$
\frac{d^{2} \mathcal{E}_{\mathrm{CTR}}}{d \omega d \Omega}=\frac{e^{2} N(N-1)}{\pi^{2} c}\left|\int d \tau d \boldsymbol{\rho} d \mathbf{v} \frac{\beta \cos \Theta[\sin \theta-\beta \sin \Theta \cos (\phi-\Phi)]}{\left[(1-\beta \sin \theta \sin \Theta \cos (\phi-\Phi))^{2}-\beta^{2} \cos ^{2} \theta \cos ^{2} \Theta\right]} f(\tau, \boldsymbol{\rho}, \mathbf{v}) e^{i \omega \tau-i \mathbf{q} \cdot \boldsymbol{\rho}}\right|^{2}
$$

Generally, the integral in Eq. (21) is too complicated to calculate analytically. In the follows, we will discuss it with some assumptions for simplification.

\section{A. A simple model}

If the distribution function $f_{\tau}(\tau, \boldsymbol{\rho}, \mathbf{v})$ can be written as the product of two independent parts,

$$
f_{\tau}(\tau, \boldsymbol{\rho}, \mathbf{v})=n(\tau, \boldsymbol{\rho}) f_{\mathbf{v}}(\mathbf{v}),
$$

the CTR spectrum (21) can be written as

$$
\frac{d^{2} \mathcal{E}_{\mathrm{CTR}}}{d \omega d \Omega}=(N-1)|\widetilde{n}(\omega, \mathbf{q})|^{2} \frac{d^{2} \mathcal{E}_{\mathrm{ITR}}}{d \omega d \Omega},
$$

where $\widetilde{n}(\omega, \mathbf{q})$ is the Fourier transformation of the function $n(\tau, \boldsymbol{\rho})$,

$$
\widetilde{n}(\omega, \mathbf{q})=\int d \tau d \boldsymbol{\rho} n(\tau, \boldsymbol{\rho}) e^{i \omega \tau-i \mathbf{q} \cdot \boldsymbol{\rho}} .
$$

We can see from Eq. (23) that the intensity of CTR could be much stronger than that of ITR in the frequency region where $\widetilde{n}(\omega, \mathbf{q})$ is notably different from zero because the electron number $N$ is usually very large. Therefore, CTR could be easily measured in an experiment.

Since $d^{2} \mathcal{E}_{\mathrm{ITR}} / d \omega d \Omega$ is independent of radiation frequency under our assumption, Eq. (23) indicates that the profile of CTR spectrum is fully determined by the function 
$\widetilde{n}(\omega, \mathbf{q})$. The angular distribution of CTR also depends on $\widetilde{n}(\omega, \mathbf{q})$ because of the relation $q=(\omega / c) \sin \theta$. As an example, we take

$$
\begin{aligned}
n(\tau, \boldsymbol{\rho})= & \frac{\left(1+\Delta \cos \omega_{0} \tau\right)}{\sqrt{2 \pi} \tau_{0}\left[1+\Delta \exp \left(-\omega_{0}^{2} \tau_{0}^{2} / 2\right)\right]} \\
& \times e^{-\tau^{2} / 2 \tau_{0}^{2}} \frac{1}{2 \pi a^{2}} e^{-\rho^{2} / 2 a^{2}},
\end{aligned}
$$

where $\omega_{0}$ is the microbunching frequency in the beam, $\Delta$ is the microbunching amplitude, $\tau_{0}$ is the duration of electron pulse, and $a$ is the beam radius. The Fourier transformation of Eq. (25) is

$$
\widetilde{n}(\omega, \mathbf{q})=\tilde{n}_{\ell}(\omega) \tilde{n}_{\perp}(q),
$$

where $\widetilde{n}_{\ell}(\omega)$ and $\widetilde{n}_{\perp}(q)$ are given by

$$
\begin{aligned}
\tilde{n}_{\ell}(\omega)= & \frac{1}{1+\Delta \exp \left(-\omega_{0}^{2} \tau_{0}^{2} / 2\right)}\left\{\exp \left(-\frac{\omega^{2} \tau_{0}^{2}}{2}\right)\right. \\
& \left.+\frac{\Delta}{2} \exp \left(-\frac{\left(\omega-\omega_{0}\right)^{2} \tau_{0}^{2}}{2}\right)\right\}
\end{aligned}
$$

and

$$
\widetilde{n}_{\perp}(q)=\exp \left(-q^{2} a^{2} / 2\right)=\exp \left(-\frac{\omega^{2} a^{2}}{2 c^{2}} \sin ^{2} \theta\right) .
$$

As seen from Eq. (26), transition radiation is always coherent in the low frequency range that $\omega \leqslant \tau_{0}^{-1}$. The intensity of CTR is also greatly enhanced near the microbunching frequency $\omega_{0}$. It is this property of the CTR spectrum that provides us a way to infer microbunching in hot electron beam; a sharp spike in the CTR spectrum should correspond to one microbunching frequency in the hot electron beam. The angular distribution of CTR is described by $\widetilde{n}_{\perp}(q)$. From Eq. (27), it is easy to see that CTR is mainly within the angle,

$$
\theta \lesssim \frac{\lambda}{2 \pi a},
$$

where $\lambda=2 \pi c / \omega$ is the the radiation wavelength. If the radiation wavelength is much smaller than the beam radius, this angle is very small. Unlike the angular distribution of ITR, which depends on the hot electron temperature, the angular distribution function is mainly determined by the Fourier transformation of the transverse profile of the hot electron beam. A measurement of CTR angular distribution could give an estimate of hot electron radius.

\section{B. A more realistic model}

In general, the distribution function $f_{\tau}$ cannot be simply written as the form of Eq. (22). This function depends not only on the generation of hot electrons in laser plasma interaction, but also on the propagation of hot electrons in the target. To study the evolution of this function, we introduce a new distribution function defined as

$$
f(t, \mathbf{r}, \mathbf{v})=\left\langle\frac{1}{N} \sum_{i=1}^{N} \delta\left(\mathbf{r}-\mathbf{r}_{i}\right) \delta\left(\mathbf{v}-\mathbf{v}_{i}\right)\right\rangle .
$$

Following the Klimontovich procedure, we can see that this distribution function should satisfy a kinetic equation with a collision term. ${ }^{35}$ Generally, the collision term in this kinetic equation is extremely complicated because numerous scattering processes can happen when energetic electrons propagates in target. Extreme electromagnetic fields can be driven when intense electron beam propagates in dense plasma. These self-generated fields can profoundly affect the propagation of the beam, and henceforth, the spectrum of coherent transition radiation. However, the inclusion of these effects will make analytical calculation impossible. As the zeroth order approximation, we just neglect any interactions between hot electrons and target matter. In this limit, the hot electrons move freely in the target. The distribution function $f(t, \mathbf{r}, \mathbf{v})$ then satisfies the kinetic equation,

$$
\frac{\partial f}{\partial t}+\mathbf{v} \cdot \frac{\partial f}{\partial \mathbf{r}}=0 .
$$

When an ultrashort laser pulse interacts with a solid target, hot electrons are generated in a very thin layer around the front surface of the target. ${ }^{8}$ The hot electrons propagate a distance which is equal to the target thickness, and then cross the rear surface. Assuming that at $t=0$ a $\delta$-like electron pulse is generated at the front surface, i.e.,

$$
f(0, \mathbf{r}, \mathbf{v})=\frac{1}{2 \pi a^{2}} \delta(z+d) e^{-\rho^{2} / 2 a^{2}} f_{\mathbf{v}}(\mathbf{v}),
$$

the distribution function $f(t, \mathbf{r}, \mathbf{v})$ is then given by

$$
f(t, z, \boldsymbol{\rho}, \mathbf{v})=\frac{1}{2 \pi a^{2}} \delta(z+d-u t) e^{-(\boldsymbol{\rho}-\mathbf{w} t)^{2} / 2 a^{2}} f_{\mathbf{v}}(\mathbf{v}) .
$$

Noticing the relation between $f_{\tau}(\tau, \boldsymbol{\rho}, \mathbf{v})$ and $f(t, z, \boldsymbol{\rho}, \mathbf{v})$,

$$
f_{\tau}(\tau, \boldsymbol{\rho}, \mathbf{v}) d \tau=\left.f(\tau, z, \boldsymbol{\rho}, \mathbf{v})\right|_{z=0} d z,
$$

we obtain

$$
f_{\tau}(\tau, \boldsymbol{\rho}, \mathbf{v})=\frac{1}{2 \pi a^{2}} \delta(\tau-d / u) e^{-(\boldsymbol{\rho}-\mathbf{w} \tau)^{2} / 2 a^{2}} f_{\mathbf{v}}(\mathbf{v}) .
$$

Substituting Eq. (32) into Eq. (21), and integrating over $\boldsymbol{\rho}$, we obtain

$$
\frac{d^{2} \mathcal{E}_{\mathrm{CTR}}}{d \omega d \Omega}=\frac{e^{2} N(N-1)}{\pi^{2} c} e^{-q^{2} a^{2}}\left|\int d \tau d \mathbf{v} e^{i(\omega-\mathbf{q} \cdot \mathbf{w}) \tau} \delta(\tau-d / u) f_{\mathbf{v}}(\mathbf{v}) \frac{\beta \cos \Theta[\sin \theta-\beta \sin \Theta \cos (\phi-\Phi)]}{\left[(1-\beta \sin \theta \sin \Theta \cos (\phi-\Phi))^{2}-\beta^{2} \cos ^{2} \theta \cos ^{2} \Theta\right]}\right|^{2} .
$$


A series of electron micropulses can be generated within the duration of a laser pulse. For example, when vacuum heating takes effect, forward hot electrons are generated once in a laser circle; ${ }^{8}$ when $\mathbf{j} \times \mathbf{B}$ heating dominates, energetic electrons can be accelerated twice in a laser circle. ${ }^{9}$ Therefore, it is reasonable to make the assumption that a series of electron micropulses is generated at $t=\tau_{1}, \tau_{2}, \ldots$. The time interval between two adjacent electron micropulses $\sigma=\tau_{\alpha}$ $-\tau_{\alpha-1}$ could be a constant if only one heating process takes effect. Obviously, this time interval is determined by the heating mechanism. In the examples mentioned above, $\sigma$ $=\lambda_{0} / c$ in the vacuum heating, and $\sigma=\lambda_{0} / 2 c$ in $\mathbf{j} \times \mathbf{B}$ heating, where $\lambda_{0}$ is the incident laser wavelength. Assuming that the properties of hot electrons (number, temperature, etc.) in every micropulse are the same, and summating the contributions from all these electron micropulses, we have

$$
\begin{aligned}
\frac{d^{2} \mathcal{E}_{\mathrm{CTR}}}{d \omega d \Omega}= & \frac{e^{2} N_{b}^{2}}{\pi^{2} c} e^{-q^{2} a^{2}} \mid \int d \tau d \mathbf{v} e^{i(\omega-\mathbf{q} \cdot \mathbf{w}) \tau} \sum_{\alpha=1}^{\Lambda} \delta\left(\tau-\tau_{\alpha}-d / u\right) f_{\mathbf{v}}(\mathbf{v}) e^{i \mathbf{q} \cdot \mathbf{w} \tau_{\alpha}} \\
& \times\left.\frac{\beta \cos \Theta[\sin \theta-\beta \sin \Theta \cos (\phi-\Phi)]}{\left[(1-\beta \sin \theta \sin \Theta \cos (\phi-\Phi))^{2}-\beta^{2} \cos ^{2} \theta \cos ^{2} \Theta\right]}\right|^{2},
\end{aligned}
$$

where $N_{b}=N / \Lambda$ is the electron number in an electron micropulse, $\Lambda=\tau_{0} / \sigma$ is the total number of micropulses, and $\tau_{0}$ is the duration of the laser pulse.

\section{One-dimensional case}

As a first step, we consider the condition that all electrons move along the $z$-direction. In this case, Eq. (33) becomes

$$
\begin{aligned}
\frac{d^{2} \mathcal{E}_{\mathrm{CTR}}}{d \omega d \Omega}= & \frac{e^{2} N_{b}^{2}}{\pi^{2} c} \sin ^{2} \theta e^{-q^{2} a^{2}} \mid \int d \tau d \beta e^{i \omega \tau} \\
& \times\left.\sum_{\alpha=1}^{\Lambda} \delta\left(\tau-\tau_{\alpha}-t_{0} / \beta\right) \frac{\beta f_{\mathbf{v}}(\beta)}{\left(1-\beta^{2} \cos ^{2} \theta\right)}\right|^{2},
\end{aligned}
$$

where $t_{0}=d / c$. We again assume that hot electrons have a Boltzmann energy distribution. The velocity distribution function is given by

$$
f_{\mathbf{v}}(\beta)=\frac{\beta}{T\left(1-\beta^{2}\right)^{3 / 2}} e^{-\varepsilon / T} .
$$

Substituting Eq. (35) into Eq. (32), and integrating over $\beta$, we obtain

$$
\frac{d^{2} \mathcal{E}_{\mathrm{CTR}}}{d \omega d \Omega}=\frac{e^{2} N_{b}^{2}}{\pi^{2} c T^{2} t_{0}^{2}} \sin ^{2} \theta e^{-q^{2} a^{2}}\left|\int d \tau e^{i \omega \tau} g(\tau, \theta, T, d)\right|^{2}
$$

where the function $g(\tau, \theta, T, d)$ is defined as

$$
g(\tau, \theta, T, d)=\sum_{\alpha=1}^{\Lambda} \frac{\left(t_{0} /\left(\tau-\tau_{\alpha}\right)\right)^{4} \exp \left[-\left(1 / \sqrt{1-\left(t_{0} /\left(\tau-\tau_{\alpha}\right)\right)^{2}}-1\right) / T\right]}{\left[1-\left(t_{0} /\left(\tau-\tau_{\alpha}\right)\right)^{2} \cos ^{2} \theta\right]\left[1-\left(t_{0} /\left(\tau-\tau_{\alpha}\right)\right)^{2}\right]^{3 / 2}} .
$$

The CTR spectrum is determined by the Fourier transformation of the function $g(\tau, \theta, T, d)$, see Eq. (36). Denoting

$$
\widetilde{g}(\omega)=\int d \tau e^{i \omega \tau} g(\tau, \theta, T, d),
$$

we can rewrite Eq. (36),

$$
\frac{d^{2} \mathcal{E}_{\mathrm{CTR}}}{d \omega d \Omega}=\frac{e^{2} N_{b}^{2}}{\pi^{2} c T^{2} t_{0}^{2}} \sin ^{2} \theta e^{-q^{2} a^{2}}|\widetilde{g}(\omega, \theta, T, d)|^{2} .
$$

When the time interval between two adjacent electron micropulses is a constant $\sigma$, we can expect that the CTR spectrum should present spikes at the frequencies of $l \omega_{0}$, where $\omega_{0}$ $=2 \pi / \sigma$ is the microbunching frequency, and $l=1,2, \ldots$ is an integer. We numerically calculate $|\widetilde{g}(\omega)|^{2}$, and plot it in

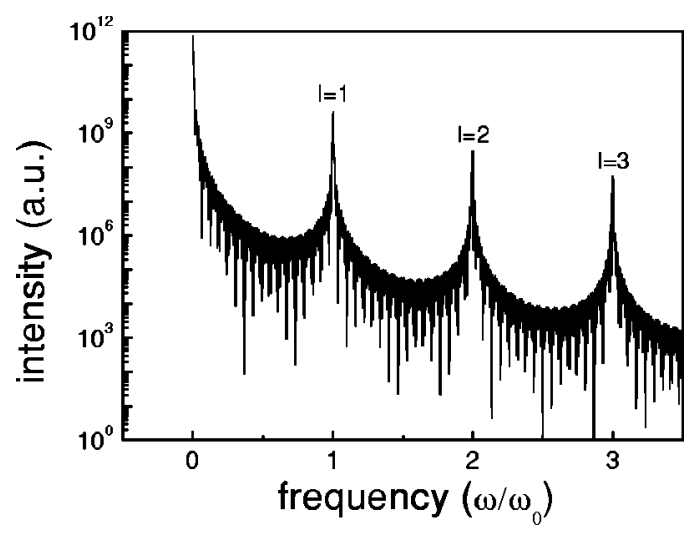

FIG. 5. Spectrum of coherent transition radiation calculated with Eqs. (37) and (38), where $T=1 \mathrm{MeV}, \theta=10^{\circ}, d=50 \mu \mathrm{m}, \lambda_{0}=1.053 \mu \mathrm{m}$, and $\Lambda$ $=142$. 
Fig. 5, in which we take $\theta=10^{\circ}, T=1 \mathrm{MeV}, d=50 \mu \mathrm{m}$, $\lambda_{0}=1.053 \mu \mathrm{m}$, and $\sigma=\lambda_{0} / c$. As we expect, the CTR spectrum reaches sharp maxima at the harmonics of the frequency $\omega_{0}$. Since transition radiation is always coherent in the low frequency limit, the spectrum also has a maximum at $\omega=0$. From Fig. 5, we can see that the intensity of the harmonics decreases very rapidly with the increase of the order $l$, even though we assume $\delta$-like electron micropulses are generated at the front surface. For example, $\left|\widetilde{g}\left(2 \omega_{0}\right)\right|^{2}$ is just about $8 \%$ of $\left|\widetilde{g}\left(\omega_{0}\right)\right|^{2}$. With the inclusion of the angular distribution, the intensity of high order harmonics should decrease more rapidly because of the factor $\exp \left(-q^{2} a^{2}\right)$ in Eq. (39).

The CTR spectrum also depends other parameters, like the temperature $T$ and the target thickness $d$, as seen in Eq. (37). Because of the finite temperature, hot electrons disperse in velocity space. The shape of electron micropulses would be broadened in configuration space due to the velocity dispersion. Therefore, the microbunching amplitude decreases as the beam propagates in the target. This can be seen more clearly from the function $N(\tau)$, which defined as

$$
N(\tau)=N \int d \boldsymbol{\rho} d \mathbf{v} f_{\tau}(\tau, \boldsymbol{\rho}, \mathbf{v})=\frac{N}{\Lambda T} \sum_{\alpha=1}^{\Lambda} \frac{\left(t_{0} /\left(\tau-\tau_{\alpha}\right)\right)^{3} \exp \left[-\left(1 / \sqrt{1-\left(t_{0} /\left(\tau-\tau_{\alpha}\right)\right)^{2}}-1\right) / T\right]}{t_{0}\left[1-\left(t_{0} /\left(\tau-\tau_{\alpha}\right)\right)^{2}\right]^{3 / 2}}
$$

$N(\tau)$ is nothing but the number of electrons crossing the rear surface in unit time. In Figs. 6(a), 6(b), and 6(c) we plot this function for $d=50,100$, and $200 \mu \mathrm{m}$ in the condition of $T$ $=1.0 \mathrm{MeV}$. As seen in this figure, the microbunching amplitude in the electron beam decreases with the increase of the target thickness. When the hot electron temperature is higher, the microbunching amplitude should also be larger if the beam propagates the same distance. This can be seen in Figs. 6(d), 6(e), and 6(f), in which we plot the function $N(\tau)$ for $d=50,100$, and $200 \mu \mathrm{m}$, but in the condition of $T$ $=2.0 \mathrm{MeV}$. The reason for this result is simple: since particle velocity cannot exceed the light speed, the electron velocity dispersion becomes smaller when the temperature becomes higher. Because of these characteristics of the function $N(\tau)$, it is straightforward to conclude that the CTR intensity would have the similar properties. Shown in Fig. 7 is the variation of CTR intensity at $\omega=\omega_{0}$ with the target

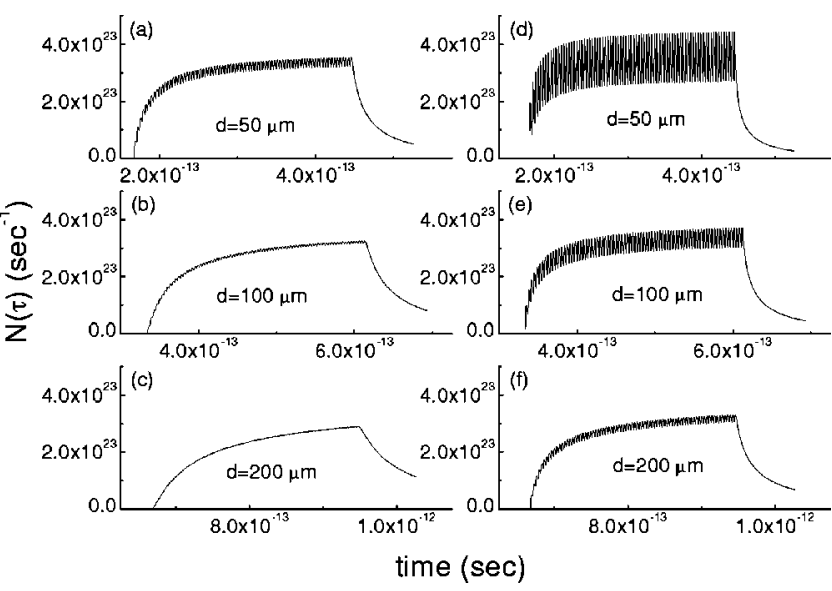

FIG. 6. Number of hot electrons across the rear surface of the target in unit time, where $N=10^{11}, \Lambda=80$, and $T=1 \mathrm{MeV}$ in the left column and $T$ $=2 \mathrm{MeV}$ in the right column, respectively. thickness and the temperature. As seen in this figure, the CTR decreases very rapidly with the increasing of the target thickness. When the temperature becomes higher, the intensity is also higher, and the decreasing more slowly with the target thickness.

In the above discussion, we do not consider any interactions between the hot electrons and the target material. In fact, electrons lose their energies, and change their moving directions when they interact with the target material. Low energy electrons are even stopped inside the target, and do not cross the rear surface. With inclusion of these effects, the decreasing rate of CTR intensity with target thickness should be larger than that shown in Fig. 7, especially when the temperature is low. In the calculation, we also neglect the influence of the front surface. When the target is extremely thin, the influence of the front surface should be considered. The finite thickness effect is relevant to the so-called formation zone, which was pointed out by Garibian. ${ }^{36}$ When the formation zone is longer than the target thickness, transition radiation from back surface is also influenced by the front surface, and the radiation energy is suppressed. Theoretical calcula-

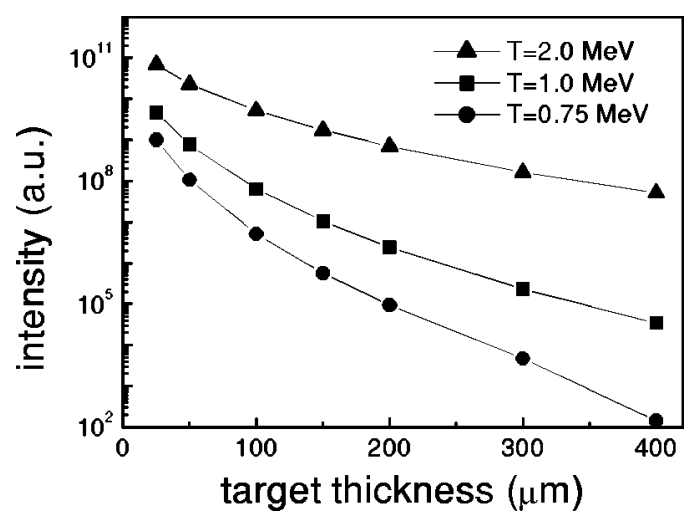

FIG. 7. Intensity of CTR at $\omega_{0}$ vs the target thickness $d$. 
tion shows that as the target thickness approaches zero the transition radiation vanishes. ${ }^{33}$ The effect of formation zone on transition radiation has been observed in experiment. ${ }^{37}$ The formation zone in a medium with a dielectric constant $\epsilon$ is given $b^{37}$

$$
Z=\frac{\beta \lambda}{2 \pi\left|1-\beta\left(\epsilon-\epsilon \sin ^{2} \theta\right)^{1 / 2}\right|},
$$

where $\lambda$ is the radiation wavelength. Since $|\epsilon| \gg 1$ in the optical region for a conductor, the formation zone is much less than the radiation wavelength. In this meaning, our calculation is valid when $d \gg \lambda$.

\section{Two-dimensional case}

Generally, hot electron beams produced in ultraintense laser plasma interactions are not highly collimated, and present rather large divergence angles. The phase factor $i(\omega-\mathbf{q} \cdot \mathbf{w}) \tau$ in Eq. (21) clearly shows that the main effect of beam divergence on CTR spectrum is the Doppler frequency shift because of the tangential velocity $\mathbf{w}$. The CTR spectrum can be broadened due to the beam divergence. We assume that the tangential velocities of hot electrons are very slow in comparison with their longitudinal velocities. Denoting $w$ $=\beta \sin \Theta$, and $u=\beta \cos \Theta$, we expand the integrand in Eq. (21) in a power series of $w$,

$$
\begin{gathered}
\frac{\beta \cos \Theta[\sin \theta-\beta \sin \Theta \cos (\phi-\Phi)]}{\left[(1-\beta \sin \theta \sin \Theta \cos (\phi-\Phi))^{2}-\beta^{2} \cos ^{2} \theta \cos ^{2} \Theta\right]} \\
\approx \frac{u}{1-u^{2} \cos ^{2} \theta}[\sin \theta+w \\
\left.\quad \times \cos (\phi-\Phi)\left(\frac{2 \sin ^{2} \theta}{1-u^{2} \cos ^{2} \theta}-1\right)\right] .
\end{gathered}
$$

For the sake of analytical calculation, we further assume that the velocity distribution is given by

$$
f_{\mathbf{v}}(\mathbf{v})=\frac{1}{2 \pi T_{\perp}} \exp \left(-w^{2} / T_{\perp}\right) f(u),
$$

where $T_{\perp} \ll 1$. Substituting the expansion (41) into Eq. (21), and using the formula

$$
\exp (-i \mathbf{q} \cdot \mathbf{w} \tau)=\sum_{k=-\infty}^{\infty}(-i)^{k} J_{k}(\omega \tau w \sin \theta) e^{i k(\phi-\Phi)},
$$

and integrating Eq. (21) over $\Phi$ and $w$, we obtain

$$
\begin{aligned}
\frac{d^{2} \mathcal{E}_{\mathrm{CTR}}}{d \omega d \Omega}= & \frac{e^{2} N(N-1)}{4 \pi^{2} c} \sin ^{2} \theta e^{-q^{2} a^{2}} \\
& \times \mid \int d \tau d u \frac{\exp (i \omega \tau) f(u) u}{1-u^{2} \cos ^{2} \theta} \delta\left(\tau-t_{0} / u\right) \\
& \times\left[1-\frac{i}{2} \omega \tau \sin \theta T_{\perp}\left(\frac{2 \sin ^{2} \theta}{1-u^{2} \cos ^{2} \theta}-1\right)\right] \\
& \times\left.\exp \left(-\omega^{2} \tau^{2} \sin ^{2} \theta T_{\perp} / 4\right)\right|^{2}
\end{aligned}
$$

The factor $\exp \left(-\omega^{2} \tau^{2} \sin ^{2} \theta T_{\perp} / 4\right)$ in this equation now clearly indicates that the CTR spectrum is broadened due to the beam divergence. The frequency broadening $\delta \omega$ due to this factor is about

$$
\delta \omega \sim \sqrt{T_{\perp}} \sin \theta \omega .
$$

When a series of micropulses is produced at the front surface, the sharp spectral spikes shown in Fig. 5 should become broader if the beam divergence cannot be neglected. Although the result (43) is dependent of the assumption (42), the conclusion that the beam divergence can broaden the CTR spectrum does not depend on this assumption. Noticing that $T_{\perp}$ is roughly the mean value of $w^{2}$ under the assumption (42), it is reasonable to rewrite Eq. (43) in a more general form,

$$
\delta \omega \sim \sqrt{\left\langle w^{2}\right\rangle} \sin \theta \omega,
$$

where $\left\langle w^{2}\right\rangle$ is the mean value of $w^{2}$.

\section{CONCLUSIONS}

We have discussed transition radiation from hot electrons generated in laser plasma interactions. The total radiation from a hot electron beam has been separated into two parts: incoherent transition radiation (ITR) and coherent transition radiation (CTR). The ITR spectrum just depends on the electron velocities. The CTR spectrum is dependent of the charge configurations as well as their velocities. In general, the formulas of the spectra of ITR and CTR are very complicated. For the sake of analytical calculation and simplification, some reasonable assumptions are made in the discussions. We find that the angular distribution of ITR can be rather flat in the hot electron temperature range of interest, and approaches that of the hot electron beam when the temperature $T$ is extremely high. The yield of ITR photons is very low. It may be impossible to obtain any useful information from incoherent transition radiation because other radiation process could overwhelm it. The CTR intensity can be many orders of magnitude higher than the ITR intensity in particular wavelength region. Hence it is easier to measure CTR spectrum in an experiment. The spectrum of CTR is determined by microbunching in the hot electron beam, from which we can infer the dominant heating process in the laser plasma interaction. The simplified model shows that the intensity of CTR decreases with the increase of target thickness, and increases with the increase of hot electron temperature. Finite beam divergence can broaden the CTR spectrum.

\section{ACKNOWLEDGMENTS}

The authors are very grateful to Professor T. A. Hall for reading the manuscript and making valuable comments.

J.Z. is thankful for the support by the Japan Society of the Promotion of Science.

\footnotetext{
${ }^{1}$ T. Tajima and J. M. Dawson, Phys. Rev. Lett. 43, 267 (1979).

${ }^{2}$ A. Modena, Z. Najmudin, A. E. Dangor, C. E. Clayton, K. A. Marsh, C. Joshi, V. Malka, C. B. Darrow, C. Danson, D. Neely, and F. N. Walsh, Nature (London) 377, 606 (1995).

${ }^{3}$ M. Tabak, J. Hammer, M. E. Glinsky, W. L. Kruer, S. C. Wilks, J. Wood-
} 
worth, E. M. Campbell, M. D. Perry, and R. J. Mason, Phys. Plasmas 1, 1626 (1994).

${ }^{4}$ R. Kodama, P. A. Norreys, K. Mima, A. E. Dangor, R. G. Evans, H. Fujita, Y. Kitagawa, K. Krushelnick, T. Miyakoshi, N. Miyanaga, T. Norimatsu, S. J. Rose, T. Shozaki, K. Shigemori, A. Sunahara, M. Tampo, K. A. Tanaka, Y. Toyama, T. Yamanaka, and M. Zept, Nature (London) 412, 798 (2001).

${ }^{5}$ E. D. Liang, S. C. Wilks, and M. Tabak, Phys. Rev. Lett. 81, 4887 (1998).

${ }^{6}$ T. E. Cowan, M. D. Perry, M. H. Key, T. R. Ditmire, S. P. Hatchett, E. A. Henry, J. D. Moody, M. J. Moran, D. M. Pennington, T. W. Pillips, T. C. Sangster, J. A. Sefcik, M. S. Singh, R. A. Snavely, M. A. Stoyer, S. C. Wilks, P. E. Young, Y. Takahashi, B. Dong, W. Fountain, T. Parnell, J. Johnson, A. W. Hunt, and T. Kühl, Laser Part. Beams 17, 773 (1999).

${ }^{7}$ W. L. Kruer, The Physics of Laser Plasma Interactions (Addison-Wesley, New York, 1988).

${ }^{8}$ F. Brunel, Phys. Rev. Lett. 59, 52 (1987).

${ }^{9}$ S. C. Wilks, W. L. Kruer, M. Tabak, and A. B. Langdon, Phys. Rev. Lett. 69, 1383 (1992).

${ }^{10}$ A. Pukhov, Z. M. Sheng, and J. Meyer-ter-Vehn, Phys. Plasmas 6, 2847 (1999).

${ }^{11}$ K. A. Tanaka, Rev. Laser Eng. 29, 238 (2001) (in Japanese).

${ }^{12}$ Y. Oishi, T. Nayuki, K. Nemoto, Y. Okano, Y. Hironaka, K. G. Nakamura, and K. Kondo, Appl. Phys. Lett. 79, 1234 (2001).

${ }^{13}$ K. B. Wharton, S. P. Hatchett, S. C. Wilks, M. H. Key, J. D. Moody, V. Yanovsky, A. A. Offenberger, B. A. Hammel, M. D. Perry, and C. Joshi, Phys. Rev. Lett. 81, 822 (1998).

${ }^{14}$ M. H. Key, M. D. Cable, T. E. Cowan, K. G. Estabrook, B. A. Hammel, S. P. Hatchett, E. A. Henry, D. E. Hinkel, J. D. Kilkenny, J. A. Koch, W. L. Kruer, A. B. Langdon, B. F. Lasinski, R. W. Lee, B. J. MacGowan, A. MacKinnon, J. D. Moody, M. J. Moran, A. A. Offenberger, D. M. Pennington, M. D. Perry, T. J. Phillips, T. C. Sangster, M. S. Singh, M. A. Stoyer, M. Tabak, G. L. Tiebohl, M. Tsukamoto, K. Wharton, and S. C. Wilks, Phys. Plasmas 5, 1966 (1998).

${ }^{15}$ R. Kodama, K. Mima, K. A. Tanaka, Y. Kitagawa, H. Fujita, K. Takahashi, A. Sunahara, K. Fujita, H. Habara, T. Jitsuno, Y. Sentoku, T. Matsushita, T. Miyakoshi, N. Miyanaga, T. Norimatsu, H. Setoguchi, T. Sonomoto, M. Tanpo, Y. Toyama, and T. Yamanaka, Phys. Plasmas 8, 2268 (2001).

${ }^{16}$ L. M. Chen, J. Zhang, Y. T. Li, H. Teng, T. J. Liang, Z. M. Sheng, Q. L. Dong, L. Z. Zhao, Z. Y. Wei, and X. W. Tang, Phys. Rev. Lett. 87, 225001 (2001).

${ }^{17}$ V. L. Ginzburg and I. M. Frank, Zh. Eksp. Teor. Fiz. 16, 15 (1946).

${ }^{18}$ G. M. Garibian, Sov. Phys. JETP 6, 1079 (1958).

${ }^{19}$ L. C. L. Yuan, C. L. Wang, and S. Prünster, Phys. Rev. Lett. 23, 496 (1969).
${ }^{20}$ M. L. Cherry, G. Hartmann, D. Müller, and T. A. Prince, Phys. Rev. D 10, 3594 (1974).

${ }^{21}$ M. L. Cherry and D. Müller, Phys. Rev. Lett. 38, 5 (1977).

${ }^{22}$ U. Happek, A. J. Sievers, and E. B. Blum, Phys. Rev. Lett. 67, 2962 (1991).

${ }^{23}$ Y. Shibata, K. Ishi, T. Takahashi, T. Kanai, M. Ikezawa, K. Takami, T. Matsuyama, K. Kobayashi, and Y. Fujita, Phys. Rev. A 45, R8340 (1992).

${ }^{24}$ Y. Shibata, K. Ishi, T. Takahashi, T. Kanai, F. Arai, S. Kimura, T. Ohsaka, M. Ikezawa, Y. Kondo, R. Kato, S. Urasawa, T. Nakazato, S. Niwano, M. Yoshioka, and M. Oyamada, Phys. Rev. E 49, 785 (1994).

${ }^{25}$ A. Tremaine, J. B. Rosenzweig, S. Anderson, P. Frigola, M. Hogan, A. Murokh, and C. Pellegrini, Phys. Rev. Lett. 81, 5816 (1998).

${ }^{26}$ J. Oostens, S. Prünster, C. L. Wang, and L. C. L. Yuan, Phys. Rev. Lett. 19, 541 (1967).

${ }^{27}$ L. Wartski, S. Roland, J. Lasalle, M. Bolore, and G. Filippi, J. Appl. Phys. 46, 3644 (1975).

${ }^{28}$ A. H. Lumpkin, R. Dejus, W. J. Berg, M. Borland, Y. C. Chae, E. Moog, N. S. Sereno, and B. X. Yang, Phys. Rev. Lett. 86, 79 (2001).

${ }^{29}$ A. H. Lumpkin, R. Dejus, J. W. Lewellen, W. Berg, S. Biedron, M. Borland, Y. C. Chae, M. Erdmann, Z. Huang, K.-J. Kim, Y. Li, S. V. Milton, E. Moog, D. W. Rule, V. Sajaev, and B. X. Yang, Phys. Rev. Lett. 88, 234801 (2002).

${ }^{30}$ S. D. Baton, F. Amiranoff, C. Anderson, D. Batani, T. E. Cowan, R. R. Freeman, L. Gremillet, T. Hall, S. P. Hatchett, M. H. Key, J. King, J. A. Koch, M. Koenig, A. J. MacKinnon, E. Martinolli, E. Perelli, M. Rabecle-Gloahec, C. Rousseaux, J. J. Santos, F. Scianitti, R. A. Snavely, and R. B. Stephens, Inertial Fusion Sciences and Applications 2001 (Elsevier, Paris, 2002), p. 375.

${ }^{31}$ J. Zheng, K. A. Tanaka, T. Miyakoshi, Y. Kitagawa, R. Kodama, T. Kuarahashi, and T. Yamanaka, Phys. Plasmas 9, 3610 (2002).

${ }^{32}$ L. D. Landau and E. M. Lifshitz, Electrodynamics of Continuous Media, 2nd ed. (Pergamon, Oxford, 1984).

${ }^{33}$ M. L. Ter-Mikaelian, High-Energy Electromagnetic Processes in Condensed Media (Wiley, New York, 1972), Chap. 4.

${ }^{34}$ C. J. Hirschmugl, M. Sagurton, and G. P. Williams, Phys. Rev. A 44, 1316 (1991).

${ }^{35}$ E. M. Lifshitz and L. P. Pitaevskii, Physical Kinetics (Pergamon, Oxford, 1981).

${ }^{36}$ G. M. Garibian, Sov. Phys. JETP 11, 1306 (1960).

${ }^{37}$ L. C. L. Yuan, C. L. Wang, H. Uto, and S. Prünster, Phys. Rev. Lett. 25, 1513 (1970). 